\title{
BLOCKCHAIN BASED AUDITABLE MEDICAL TRANSACTION SCHEME FOR ORGAN TRANSPLANT SERVICES
}

Gasim Alandjani

Assistant Professor, CSE Department, Yanbu University College, Royal Commission Yanbu (Saudi Arabia). E-mail: alandjanig@rcyci.edu.sa

Recepción: 26/07/2019 Aceptación: 18/09/2019 Publicación: 06/11/2019

\section{Gitación sugerida:}

Alandjani, G. (2019). Blockchain based auditable medical transaction scheme for organ transplant services. 3C Tecnología. Glosas de innovación aplicadas a la pyme. Edición Especial, Noviembre 2019, 41-63. doi: http://dx.doi.org/10.17993/3ctecno.2019. specialissue 3.41-63

\section{Suggested citation:}

Alandjani, G. (2019). Blockchain based auditable medical transaction scheme for organ transplant services. 3 C Tecnología. Glosas de innovación aplicadas a la pyme. Special Issue, November 2019, 41-63. doi: http://dx.doi.org/10.17993/3ctecno.2019.specialissue3.41-63 


\section{ABSTRACT}

Internet of Things have brought exciting changes in the social norms, work environments and the prospects for future generations. These devices (Things) have already changed the way our networks are used for communication. With the introduction of machine to machine communication (M2M), where devices communicate without human involvement to perform routine day to day tasks. These tasks on one hand, include services that provide convenience to the device owners such as setting off alarms, acting as personal assistant for reminders, keeping track of daily activities etc. On the other hand, there are certain tasks where these devices perform Transactions on behalf of the owners i.e. financial transactions/ online ordering etc. These transactional tasks have significant legal implications if some problem / dispute arises due to such action performed by these devices on behalf of the owners. To ensure that these interactions take place under observation of the owners and to keep track of their occurrence, there is a need to keep record of all such communication. We propose use of Blockchain for tracking all these transactions without compromising secrecy of data by keeping its integrity intact for medico-legal requirements and prevent risk of fraud.

\section{KEYWORDS}

IoT Security, Blockchain, Organ Donation, Smart Health, Nodes, Mining, UPRL, IDoT, M-IOT, P2P. 


\section{INTRODUCTION}

Since the introduction of bitcoin cryptocurrency by Satoshi Nakamoto (2019) and implementation of its underlying technology called Blockchain, many diverse applications of Blockchain have been proposed by the researchers. Apart from the traditional use of Blockchain that provides distributed, secure, trustable and anonymous ecosystem to allow cryptocurrency transactions, its use in the field of medical record management, counterfeit drugs detection and fraud prevention is a new area of research. As the economic disparity between the developed and developing countries is increasing day by day, there is a continuous upsurge in the demand of organs from third world countries to the advanced countries. This has increased the potential of human organ trafficking and poaching for financial benefits. Despite, efforts by the governments to formalize the laws regarding organ donation to prevent mistreatment of donors, there is a dire need of a secure, traceable and distributed organ management and distribution system. In this paper, a Blockchain based scheme is proposed to allow auditable medical transactions to prevent organ trafficking and tracking of legitimate organ donors \& recipients. This will open a new field of Smart Health services based on Blockchain to safeguard the rights of medical professionals and patients. With the advancement in science and technology, new techniques have been introduced and humanity is getting benefit from these latest innovations especially in medical field there came a revolution when transplantation of human body part took place in recent history. Organ transplantation has been used as a novel medical procedure that allows grafting of an organ from donor's body to replace a damaged or missing organ of the recipient. Due to its effectiveness and importance as a lifesaving technique, there is an ever-increasing number of patients waiting for transplant operations. Patients are no more on the mercy of fate to live their lives with organ disabilities. Now they can easily change them with healthy body parts of another person. With this level of advancement, it has become a routine task in some prominent hospitals to transplant organs. According to the American Transplant Foundation, 113,000 people in the United States are on the waiting list to receive an organ as of March 2019. Every 12 minutes a new name is added to the list and an average of 21 persons per day die due to a lack of organ availability. 
According to the Department of health $(\mathrm{DoH})$ approximately 2,000 new waiting patients are added into waiting list for organ transplantation in US only, Corneas, kidneys, intestines, liver, bone marrow and lung, are the most common transplant needs now a days (Decoded Science, 2019). WHO is working on this but the most authentic data is from The U.S. DoH \& Human Services related to complete Organ translation process starting from its procurement from black market, selling to targeted customers and then eventually its transplantation Network have publish a report, according to this report there are approximately 121,333 organ transplant are awaiting and kidney is having a very high number with awaiting 100,402 patients of kidney recipients. Legally only 30,970 transplants took place in 2015 more and less the same number was issued for 2014. WHO have reported about illegal import of organs by many developed countries all the way from under developed countries, USA is one of them and as per internet statistics patients are able to get transplant of different organs starting from range of $\$ 70$ to till $\$ 160,000.00$ as per nature and demand of organ. More than 120650 patients waiting for organ transplant in USA. There always more demand for fresh new organs which has created a perfect condition for corrupt medics to exploit organs from those who are less privileged and then sell them to needy people who have much and ultimately in this whole process they get huge margin of profit (List25, 2019). According to the WHO, an estimated 10,000 black market operations are being carried out by illegal purchase of kidneys. Even in this current era of modern technologies still in some part of the world many people have become victims of forcefully/involuntary organ donation with the help of black market of pharmaceuticals. There are many international organizations working to eliminate this illegal traffic of organs which include Organ failure solutions, ESOT and Organs watch, the average male organ donor is about 28.9 years old having a low average annual income of $\$ 480$ on the recipient side the average age for a male is about 48.1 years and an annual average income of $\$ 53,000$. Considering current situation, Medical experts have a view that based in long queue due to lengthy procedure for organs retrieval from dead bodies, there is a need to legalized organ donation by implementing strict and transparent policy in place. Lack of legal framework on organ donation and poor law enforcement helps black market where they offer little incentives to needy people and take away their 
organs. A great number of patients go to China, India or Pakistan for surgery can pay up to $\$ 200,000$ for a kidney to traffickers who harvest organs from vulnerable, desperate people, sometimes for as little as $\$ 5,000$. Estimates state that kidneys make up $75 \%$ of the global unlawful trade in organs and because of the rising rates of diabetes, high blood pressure and heart problems the demand for kidneys far outstrip supply (Whoin, 2019). According to the Bulletin of the World Health Organization, Volume 85, Number 12, December 2007, 955-962, Many websites are online that allow patients traveling abroad to purchase organs for transplants. The transplant package range in China was as follows: Kidney (\$70 000), Liver $(\$ 120000)$, Pancreas (\$110 000), Kidney and Pancreas (\$ 160000$)$ while on average, same package of Kidney transplant in Pakistan costed around US\$17000 (Decoded Science, 2019). As there are many black markets in every walk of life so is the case with human organs and there exist a black market where you can buy livers, lungs, Kidney and even hearts based on worldwide failure rate there is more demand for kidney in black market as compared to any other organ. According to the World Health Organization, this is the reason that approximately 7,000 kidneys are illegally harvested sent to their required destination by traffickers as per their black-market network. The prices for the same organ vary from country to country based on its demand and supply conditions. If we calculate worldwide data for price of human kidney then an average buyer spends $\$ 150,000$ to purchase it from black market depending on his access to any particular black market. On the other side the average donor only gets an average amount of $\$ 5,000$. This huge difference in prices facilitate the middlemen (commonly known as "organ brokers") to get huge margin of profit during this transaction. In this black-market business, the profits are enormous, and money is used to lure many brokers and even doctors and supporting paramedical staff and eventually they just cannot resist. In 2010 WHO estimated about 11,000 organs were obtained on the black market. WHO based on statistical data and associated findings also claims that organs are being sold in black market and continuous supply for organs is available which make to sell organ in almost every hour 24/7/365. 


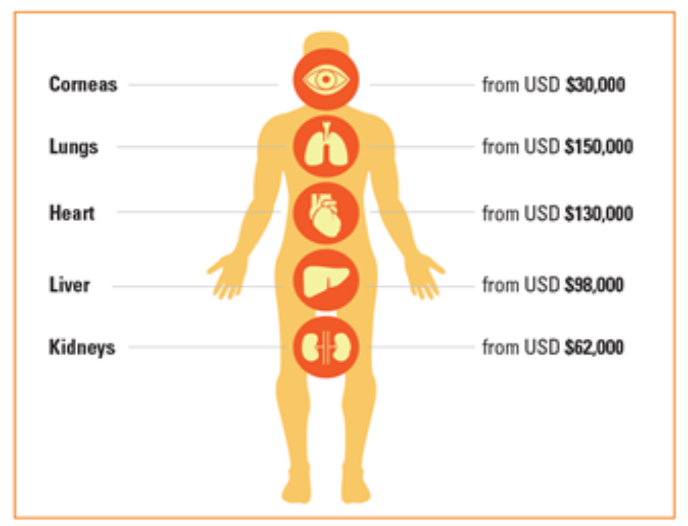

Figure 1. International black-market rates for organs.

On a world map you can find many geographic locations where human organs are being sold to potential recipients.

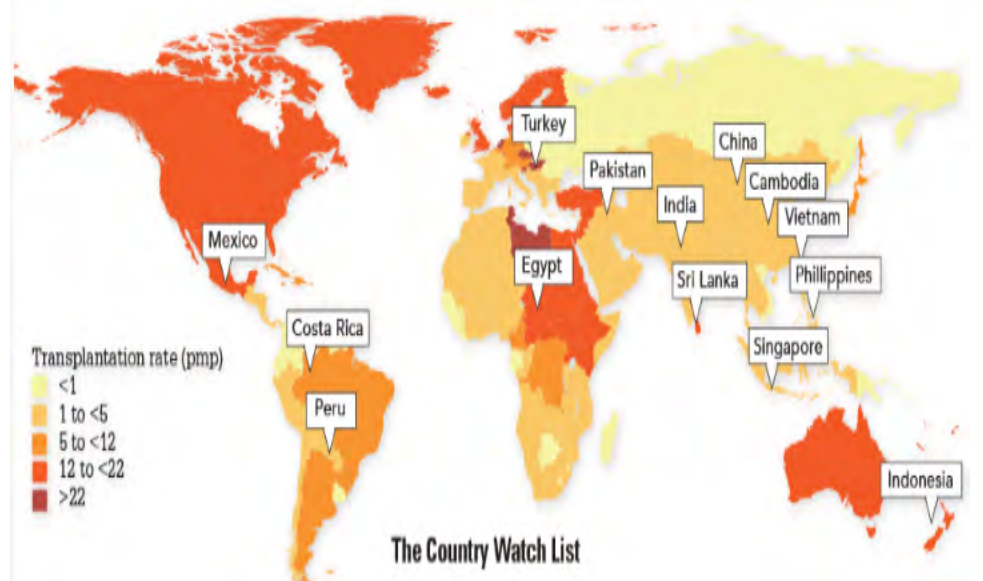

Figure 2. Global distribution of Living donor transplantation Activity -2017. Source: (Global Distribution, 2018).

Many international conferences are hosted by WHO and the February 2017 Summit was one of them. This summit was organized on topic of Organ Trafficking in Vatican City, this summit also shared research and findings on the state of the organ trade. Even with this much technology advancement still the extent of organ trafficking and their transplantation with success and failure rate is not precisely known. Which further complicate human trafficking due to demands of their organs in black market. 
In order to effectively combat organ trafficking there is need to increase its visibility by complete tracking record of organs starting from donor till recipients, every step should be documented with some reference number, fully hashed with time stamp and unchangeable. This will help to reduce this kind of organized crimes, there should be effective engagement of public-private partnerships including healthcare and insurance companies for complete success to get rid of these kind of crimes (Alarming Facts, 2019).

The solution lies in secure, track able recipient/donor profiles and historical integral information system. Which ultimately means ensuring security of patient's medical data and personal information is of utmost importance for any application in medical domain. Consider a patient with wearable sensors that record any change in the patient health-such as-high blood pressure, heart attack, faintness, blood sugar level etc. The sensors send the information to cloud storage, where analytical procedures are built, and other data related to the patient such as his medical history is already saved. Real-time data from the sensors and the historical data from the cloud is used to conduct classification and predication of the patient's case. These findings are sent to a monitoring doctor that can get alert of the changes in condition of that particular patient. It works in a reverse order to keep track of all the all phases during from start till current status of transaction. Before going to implement Blockchain solution we present Blockchain internal architecture, its working and essential components which play important role in building a secure Blockchain solution for organ donation through a track able, secure and unchangeable solution.

\section{BLOCKCHAIN HISTORY AND ARCHITECTURE}

The history of Blockchain Technology started with in 2008 when Nakamoto introduced E-currency platform which is commonly known as cryptocurrency for Bitcoin. In technical terms it is considered as a distributed database for storing a constantly developing detail of records stored in the form of list in the same database also known as blocks. A Blockchain is redundant yet verified list of records which manipulate as result of different transactions in a peer-to-peer network of nodes, 
with the condition that each node will be having a copy of all records in the form of a mirror image of the whole database. Blockchain devices connect to each other in sequential list of blocks since each block except first block which is also known as the Genesis Block in blockchain terminology, contains information about and also a link to the previous block and considered as a hash of the previous block. Every block in a Blockchain is also containing a time stamped which in a way differentiate them from other blocks. Blockchain provides a decentralized peer-to-peer platform for tracking all transaction related to any process without the need of a trusted third party. Using Blockchain for medical domain is a relatively new area of research. In this paper, we propose using Blockchain for tracking organ donation process for secure, safe and auditable operation. Following series of figures (3-6) are explaining step by step procedure starting from formation of block its entry in the chain and then its functionality.

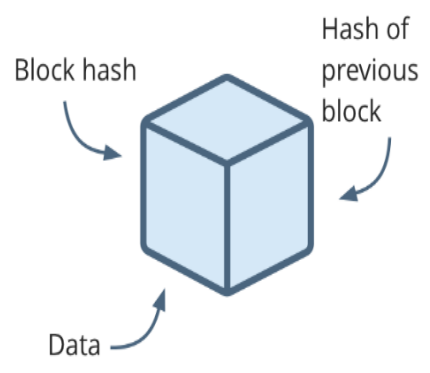

Figure 3. Formation of a Block.

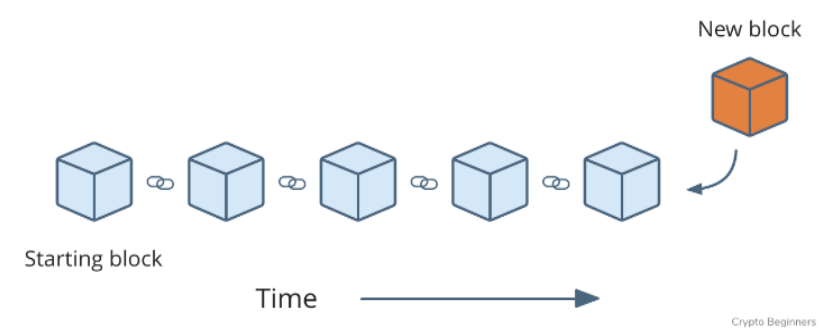

Figure 4. Adding of Blocks in a chain series. 


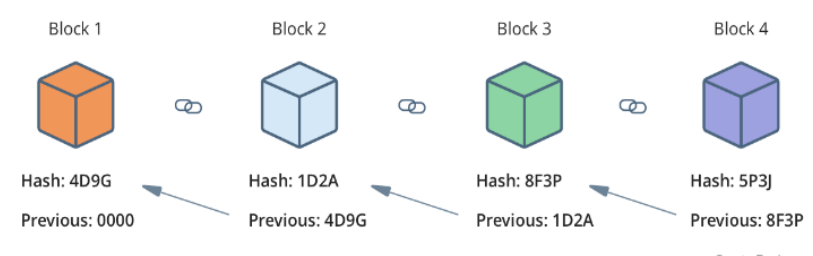

Figure 5. Block Addition with Hash Numbers.

Above figure 5 is showing how blocks are connected to other blocks and keeping its own hash tag and pointing to hash tag of previous block it will reject the entry into block if hash is not matched as given in Figure 6 below.

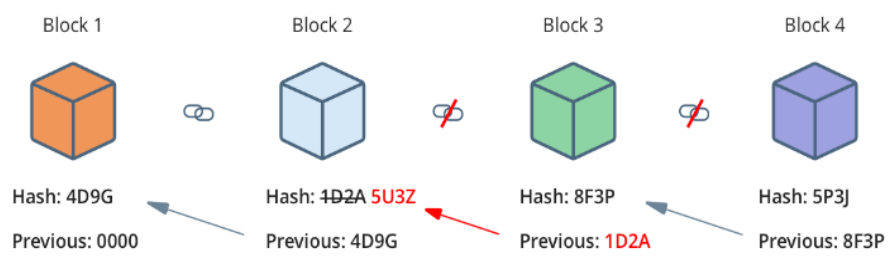

Figure 6. Denial of Block based on incorrect hash Number.

Blockchain works as distributed ledger using blocks of data. Each block contains multiple transactions by hashing them and each transaction will have the network timestamps on it. When a new block is created it will be added to the previous written blocks and the process goes on and on to complete the chain of blocks. Then all blocks and details of all previous transaction are stored in the user disc storage named node. The information held inside of nodes will be used to verify new transaction so new nodes will be added to the user chain or will be aborted. This technique is called mining which insures proof-of-work feature (Diawar, 2019) Blockchain technology can be utilized in many fields of life i.e. Supply chain, Real Estates, Automobiles, Digital identity, Governance etc. Let's consider a use-case of Blockchain in healthcare services. 


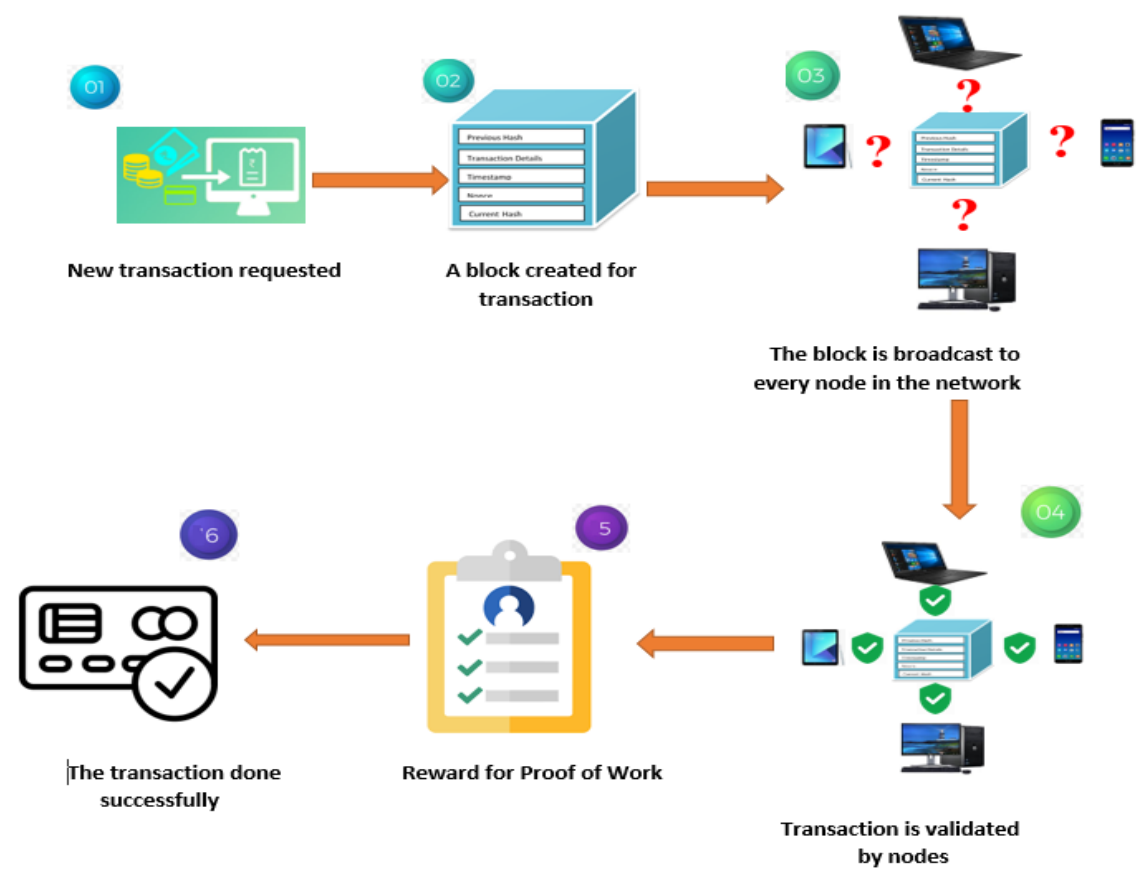

Figure 7. Complete Workflow diagram of Blockchain.

\section{USE OF BLOCKCHAIN IN SERVICES}

Blockchain technology is incorporated in various domains due to its compelling benefits in improving systems efficiency, transparency, safety and integrity. A wide range of domains are utilizing Blockchain approach including health, financial, manufacturing, business, governmental, and educational domains. With respect to healthcare domain, several applications and ongoing systems are utilizing Blockchain for improving medical record management, enhancing insurance claim process, and accelerating clinical research (Ivan, 2016). Adopting Blockchain for storing patient's health record data and management of medical records will enable patients to control access to their healthcare data. This will eliminate the need to acquiring copies of the healthcare data or sending data to another healthcare provider. Numerous companies are involved in adopting Blockchain technology such as Healthcare Data Gateways (Snow, Deery, Kirby, \& Johnston, 2015). Guardtime, a well-known company, is using a Blockchain-based system to secure 1 million 
health records in Estonia (Williams-Grut Technology Behind Bitcoin, 2019). Due to the transparency and immutability of Blockchain technology, it is used in some of the governmental services. For example, Danish political party deployed the first Blockchain voting application for internal elections in which every vote is recorded in a secure environment and stakeholders can participate and observe other votes (Millet, 2019; Ojo, n/d). In financial domain, numerous applications have been introduced for employing Blockchain technology. One of the most popular Blockchain-based application in financial area is cryptocurrency. It guarantees a secure environment for financial transactions in virtual currencies such as Bitcoin, Ripple, Litecoin, and Monoro. Various Blockchain applications are related to stock markets services such as securities exchange, smart contracts, trading and settlement, and payments and remittance. Generally, these applications aim to simplify and speed up the traditional process. Even in educational domain, Blockchain technology is used in different scenarios. Several educational institutions have adopted Blockchain for various problems. For example, the National University of La Plata (UNLP) developed a Blockchain-based framework that verifies students' academic achievements and accordingly issues the diploma. In 2015, a school in San Francisco started to utilize Blockchain in order to assist employers to validate the academic credentials. Shahbaz Pervez (2018) explains that in current technological era, everything revolves around technology and technology rotates around cloud applications and communication over internet. Security of data and associated applications is of great importance which can result into drastic results if there is any negligence from programmers over the security issues, SDLC cycle.

\subsection{USE OF BLOCKCHAIN IN HEALTH INDUSTRY}

Today's healthcare industry is awfully multifaceted with a range of stakeholders, which is a public private partnership of government agencies and private organizations including insurance companies, pharmaceutical manufacturer and end users of these medical products. There is a huge gap between healthcare providers and end users because of non-transparent information exchange which ultimately affects the entire healthcare including medical system. In the healthcare industry, collaboration 
for stakeholders is always difficult based on their particular business goals, intentions and even approached to address all these issues and their solutions. The goals are not properly aligned which result into fragmentation and unproductive services for patients. Based on varied stakeholders and their business goals, conflict of interest is inevitable, there is a great need that healthcare system should have effective collaboration, transparent processes and track able communication channels. But in real world still there need to fix some data handling and communication flaws with appropriate flow of information and its process audits which are extremely costly and eventually resulting in slowing down of healthcare services. Governments and health Insurance companies are major stakeholders which participate in drafting and implementing healthcare policies with the help of paramedics. Blockchain helps in several ways to ensure the integrity of data with proper tracking details of all transactions right from start of Blockchain enabled UPRL. Government agencies and international organization working in health sector, including WHO could have the liberty to access real-time data from any hospital for research purposes and to study different used cases based on this data which would help to determine the health conditions of people living in specific area and even in a broader case over all national health condition of a country. It will also help government agencies in tracking of any infectious disease, their causes based on lifestyle of people and to provide better health services with effective and more transparent way. This will also help the insurance companies to solve the long-awaited problem by tracking all transactional records which will ultimately prevent frauds, which is costing US only about $\$ 68$ billion per year ( The National Heath Care Anti-Fraud Association, 2018). Use of Blockchain technology enables scientists, research organization, Universities and even pharma companies. Through this technology they will get better access to data without having fear for compromise on security of data and privacy of patients which will eventually contribute for research. Paramedic's staff including doctors and other medical staff including doctors and nurses will also get advantage of Blockchainbased systems in performing their day to day routine tasks with more efficiency and peace of mind. By accessing Universal Patient Record ledger, will reduce ambiguities related to recovery or reaction of any medicine on any particular patient or group of patients in some particular situation. If the UPRL is also connected with latest 
technology gadgets including wearable technology and Medical IoT (M-IOT) devices, healthcare professionals can easily record first response, set action plans and emergency procedures with less error, by reducing number of casualties.

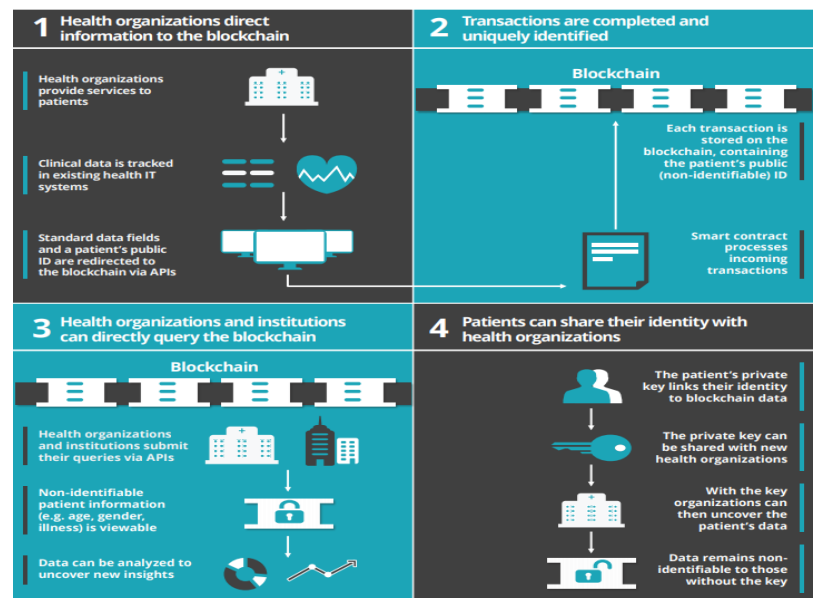

Figure 8. Illustrative Healthcare Blockchain Ecosystem. [10]

Above Figure 8 is showing overall Blockchain enabled healthcare ecosystem in four stages, starting from healthcare organizations, through secure storage and ensuring integrity of data and to end users by ensuring secure and temper free communication from start to end of a medical process for a patient.

As far as cost is concerned, Use of Blockchain based services will also help in decreasing response time and overhead costs by eliminating paperwork which unnecessarily consume lot of time of doctors and nurses in paper work and administration tasks (i.e. starting from patient entry till discharge) reduction in these tasks will eventually help patients for gaining more attention of doctors, they can also utilize this time in some innovative work for betterment of patients. With the use of Blockchain, all stakeholders including patients, their relative's and even hospital staff will be having more confidence on doctors, hospitals and even overall healthcare system related to originality of medicines and other medical products by placing a complete tracking system in Blockchain enabled network. Patients enjoy liberty of having access to their personal information by monitoring medical history by accessing laboratory reports and doctors' recommendations for curing from a specific medical condition. This will 
also ensure transparency of whole process and ultimately it will turn health insurance companies and governments into a patient-centric, traceable and more transparent system. It will also prevent fraud, especially in international market by introducing blockchain based supply chain management system. This is the most important issue that we are going to address in this research, resulting in fraud prevention in organ donation through transparent and traceable system of Blockchain.

\section{PROPOSED SYSTEM: USE OF BLOCKCHAIN IN ORGAN DONATION}

Based on its practical outcomes, trust in results, integrity and almost foolproof security in Blockchain there is an enormous increase in the use of Blockchain technology in healthcare. The importance of Organ donation cannot be ignored in healthcare system. It is a process of giving of an organ and tissue to support someone that needs transplant. Which ultimately can protect or transform the life of a person. The process of organ donation needs to be efficient, secure, distributed, trackable and immutable that can only be achieved if Blockchain technology carefully used for the processes.

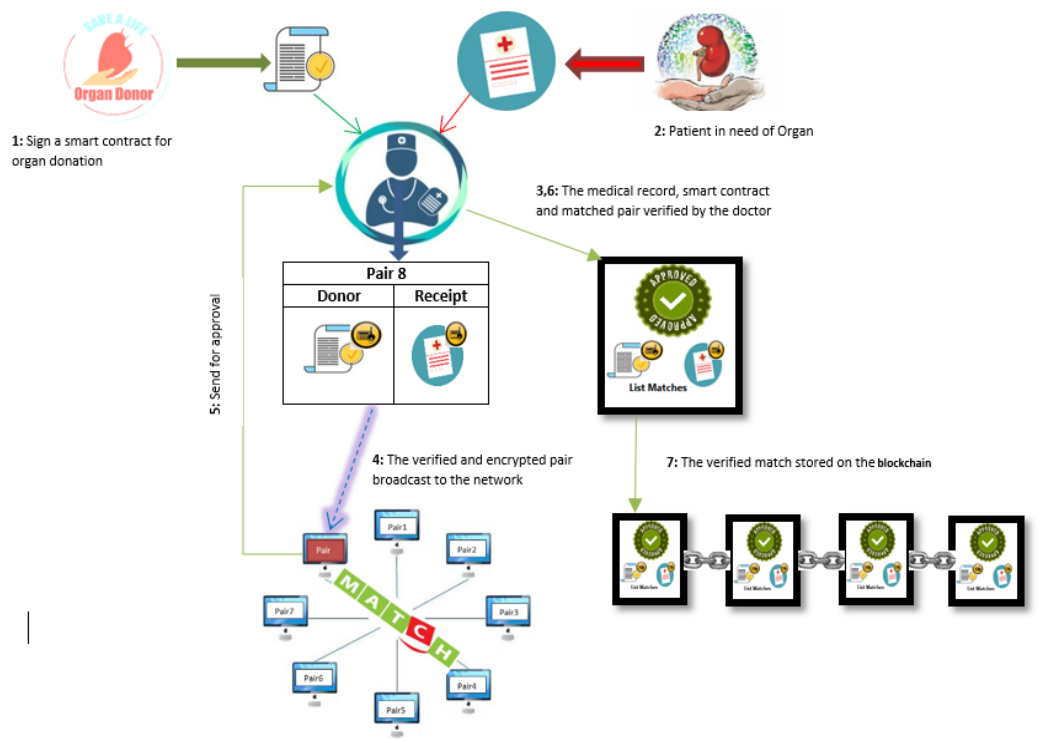

Figure 9. Use of Blockchain in Organ donation Process. 
A typical use-case for organ donation and matching process is depicted in Figure 9. The donor and receipt record can be seen as a global database, based on Blockchain technology, secured with cryptographic tools. Using the Blockchain technology in organ donation, the matching process increases chances of promptly finding an appropriate match, while being completely secure. Confidentiality and privacy can be achieved using the encryption tools and techniques. Also, the authenticity can be done using the approved digital certificates issued by authorized healthcare bodies.

The use case functions in real-time and is distributed. The process of organ donation as shown in diagram (Figure 9) starts with the donor sign a smart contact for organ donation and the patient/receipt files a request for transplant. Both the documents are verified and hashed from authorized healthcare doctor, the doctor make a verified mismatching pair and broadcast over the network pair. The network finds a match and send the match to doctor for approval, if the match is perfect the doctor give the approval and next step is to generate the hash and if doctor generate a hash, the verified matched pair will then become the part of Blockchain which cannot be altered or tempered at any later stage. Doctor and healthcare professionals receive all the information needed to arrange the operation logistics.

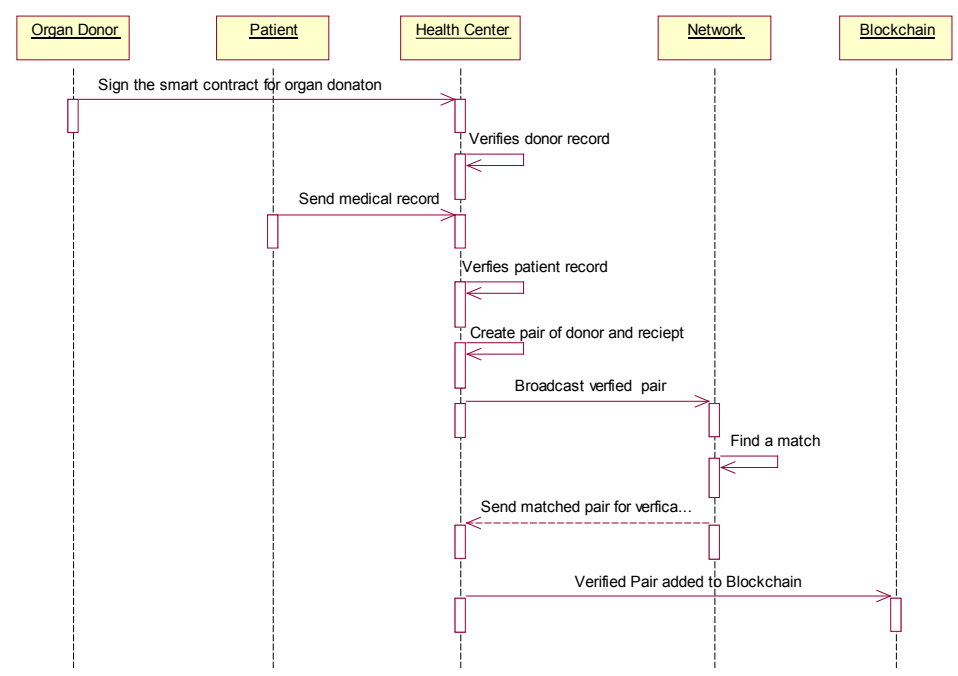

Figure 10. Sequence Diagram for Organ donation system. 
The interaction and exchange of information among Organ donor, patient and healthcare unit all are secure using the smart contact and hash tagging capabilities of Blockchain technology. The verified matched pair when broadcasted to Blockchain, this action become immutable and hence give all the involved parties the integrity, security and conformance of available information. The health unit here can act as a miner All the steps have been completed in a predefined sequence which have been shown in above sequence diagram in Figure 10 which initiates process from the agreement between Organ donor and patient (which is also known as receipt of organ), one both entities agree and sign document then its forwarded to healthcare unit for verification of medical records and sample matching process. If samples are not compatible then the request will be discarded and with the note from medical experts not to proceed with this process based on their expert opinion supported by technical details on this particular case, but if the samples are compatible and are best fit for the patient then request will be forwarded to network and with digital signatures and after approval of that request it will become part of Blockchain which finalize it and no further modification can be done after this step. Collaboration between different entities which are part of this organ donor process have been shown in in a collaborative diagram as shown in Figure 11 which is where medical experts verifies medical records of donor as well as for patient and create pair of donor and recipient, After creation of this pair and verification they broadcast it on network for finding best possible match shows the initiation of collaboration process by organ donor, which signs a contract with Health Center for organ donation.

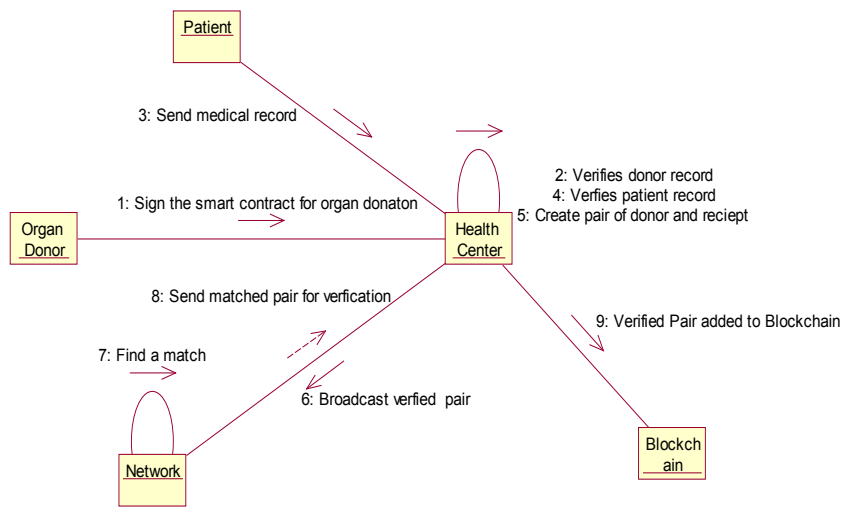

Figure 11. Collaboration diagram for Organ Donation System. 
Once they get best fit match, they further send that match for verification and after successful verification from health center this will send to Blockchain to make it part of Blockchain now this record will be considered unchangeable with integrity intact for all kind of transactions on this process.

\section{RELATED WORK}

Significant work has been done in the context of Blockchain and security. This section surveys Blockchain-based security solutions by addressing some security issues and provides an understanding for current research trends. Furthermore, some recent research work has been carried out to make Blockchain technology appropriate for IoT enabled medical equipment and hardware and software-based security issues. The physical issues are related to computational power and memory. The softwarebased issues are related to privacy, defense against malware, authentication and the regularization of governmental /organizational policies as per international technological norms, and monitoring. Main issue which is considered as one of the biggest limitations in creating a decentralized topological design is mutual trust of all the entities participating in this process. The main idea behind Blockchain is its beauty of decentralization which means it's not solely dependent on any single authority, and is not prone for having a single point of failure by attacking that particular target with the intention to target integrity of data, since complete mirror image of data is stored in all nodes of a peer to peer $(\mathrm{P} 2 \mathrm{P})$ network. Which ultimately making it difficult for attackers to fulfill their goals. However, if an attacker somehow manages to get control of several nodes in a P2P Blockchain network, with mining access permission to mining nodes, then possibility to compromise integrity of data in the attacked Blockchain. A Blockchain is considered as a distributed database for storing an uninterruptedly growth of listed records called blocks. The public key is used to identify a Blockchain user. Each Create, Read, Update or Delete operation which is also known as CRUD operation on an IoT enabled Systems, which can be registered as a transactional update/ record in a Blockchain based blocks of record. The distributed and autonomous liberty of a Blockchain make it an ideal component in IoT security enabled environment. The main limitation for resource limitation 
in IoT devices their low processing and storage capabilities due to nature of their routine tasks. There is no Blockchain-based IoT security solution available with a claim to foolproof security against threats and all kind of attacks by fulfilling all security needs in proposed standard security procedures. As of now there is no such system currently in operations, all these are just theoretical findings which might be implemented in future. Whereas a foolproof operational security model for an IoT system can be combination of standard information security procedures and Blockchain-based security.

As per normal practice is going on there are many known and unknown security threats while distributing medical information which include the forgery, no tracking of records updates. (Chen, 2018). Proposed blockchain based medical information sharing model to cope with such security threats and constructed a medical information sharing model based on blockchain by implementation to verify satisfaction for security requirements for medical information. Which confirmed that the blockchain based medical information sharing model can provide the reliability and traceability of medical information for prevention of forgery and modification in transaction or alteration of medical information. (Kuo, Kim, \& Ohno-Machado, 2017) summarize existing Blockchain protocols and application domains of Blockchain technologies in Internet of Things (IoT) networks. The application domains of Blockchain technologies in IoT discussed in this paper are: Internet of Vehicles, Internet of Energy, Internet of Cloud, and Edge computing. The authors also classify the threat models that are considered by the Blockchain protocols in IoT networks, into five main categories, namely, identity-based attacks, manipulation-based attacks, cryptanalytic attacks, reputation-based attacks, and service-based attacks. The study also outlines both open questions and research challenges that could improve the capabilities and effectiveness of Blockchain for the IoT. The paper has summarized research for Blockchain-based IoT security and privacy in terms of Blockchain model, security model, Goal, Performance and Complexity. To implement Hyper-Ledger Fabric in a smart-IoT environment to assess the validity of the communicating devices whether normal or malicious i.e., to assure users of the integrity of the data from any device. There is history of well- 
known attacks such as DDoS attack. Shen (2019) have proposed a security solution for IoT-eHealth system by presenting a context-aware and self-adaptive security model to mitigate the traditional problems, including limited context with optimal autonomous response. The IoT-eHealth multivariate and complex concepts have been simplified using Ontology technique. The system structure shows procedure from context collection till feedback. The system comprises of three layers Device Layer, Security Management Layer and Cloud Layer (Waqas, 2019). At Device Layer Local Context Manager collects device native context. Context Manager Parse, refine and transform the monitored context in in Security Management Layer. Context Agents are utilities to capture communication pattern for in/out-bound of devices. Context Analyzer with support of Machine Learning models analyzes the possible risks. Alert Generator converts any security context to an alert that will be responding by Context Adapter for optimal security according to Adaptation Rules. Evaluator will assess all the selected mechanism using a score to decide for new optimal security configuration, which can be communicated by Messenger. The proposed architecture aims to fulfill the following objectives: Holistic security, SelfAdaptation and context awareness, Open connectivity and Minimize decision delays. The proposed model can manage and deal with dynamic and multifaceted traits of a system. In fulfilling future regulatory demands, choosing Blockchain technology would be a wise selection by technology companies (Legal Issues and Regulatory Developments, 2018). Still some more research is required to design of Blockchain supportive and IoT enabled devices, good news is that European Parliament has approved new regulations and adopted them in some governmental services (Alvarez, 2018). General Data Protection Regulation (GDPR) and the Directive on Security of Network and Information Systems are approved legal acts for guiding the development and maintenance of information systems. There are minor differences in GDPR between these members, but it lays the foundation for a unified digital single market within the European Union by implementing its major theme. The NIS will likely be adopted by all states with minor modifications as per state laws of different European union member states but overall it defines a minimum level to implement security responsibilities for information systems which is the backbone of Blockchain enabled systems. There is another regulation related to general data 
protection regulation which splits the role of the controller and processor as totally separate legal entities. The GDPR also help to defines data in two categories which are known as personal and sensitive data under special category. An identifiable natural person is one who can be identified by reference such as a name, location of data, an identification number, or special category factors. IOT enables systems are not new in practical world but still security challenges are there which are still seeking attention to solve them with minimal security and privacy breach. In case of IOT enabled networks there are some findings which shows that they have potential to securely work without human supervision. There are also some limitations to use Blockchain in security solutions for IoT enabled systems (i.e. limited processing \& storage capabilities, absence of up to date firmware due to non-availability of rapid firmware updates. As a result, when Blockchain nodes increase in its blocks its ledger automatically grows. To alleviate these limitations, some alternate mining procedures should be opted and only a device-dependent transaction ledger should be maintained in case if device is too resource constrained.

\section{CONCLUSION AND FUTURE WORK}

In this article, we emphasized the prominent and vital impact of Blockchain technology and its effective use in the entire healthcare sector (i.e. from manufacturer till patients) by highlighting the hurdles in facilitating a clear exchange of information from origin till its destination and also discussed the role of Blockchain technology in solving problems related to grey market of organ donation and other related issues to help every stakeholder for making better-informed decisions. Based on our findings during this research we can conclude that use of Blockchain is going to be a must for any critical medical services and their tracking. As Blockchain can provide a temper-proof feature of recording every transmission in the digital communication, it will allow the saved data to be viewed publicly with no alteration risk by accomplishing decentralized agreements. Since health information of any person is very personal stuff which cannot be shared publicly so there is great need to address secrecy of individual health records. As medical records of a person have more fields of information so there is a need to address these issues because 
Blockchain was primarily designed for small data (bitcoins) to store and process so there was no issue related to data storage due to size and nature of this data while on the other hand storing health information systems consisted of huge information where the stored data are enormous, sensitive and it also needs further study to keep track of patient condition. It should be dealt by storing data in traditional database due to huge quantity and privacy of data and to track this hash references should be stored in the Blockchain which allow an authenticate access to the database keeping its authenticity intact. Further research can be done in this area to ensure security of data stored in the conventional databases and their linking with the Blockchain.

\section{REFERENCES}

Alarming Facts. (2019, Feb 12). Retrieved from: https://list25.com/25-alarmingfacts-about-organ-trafficking

Alvarez, M. (2018). A Comparative Analysis of Cryptocurrency Regulation in the United States, Nigeria, and China: The Potential Influence of Illicit Activities on Regulatory Evolution. ILSA Fournal of International \& Comparative Law, 33-56.

Ghen, Y. (2018). Blockchain-based medical records secure storage and medical service framework. Fournal of medical systems, 5 .

Decoded Science. (2019, February 07). Retrieved from: https://www. decodedscience.org/organ-harvesting-human-trafficking-black-market/56966

Decoded Science. (2019, March 7). Retrieved from: https://www.decodedscience. org/organ-harvesting-human-trafficking-black-market/56966

Diawar, N. R. (2019). Blockchain: Securing Internet of Medical Things (IoMT).

Diawar, N., Rizwan, M., Ahmad, F., \& Akram, S. (2019). Blockchain: Securing Internet of Medical Things (IoMT). International fournal of Advanced Computer Science and Applications, 82-89.

Global Distribution. (2018, december 17). Retrieved from: Global distribution of Living donor transplantation Activity -2017 
Ivan, D. (2016). Moving toward a blockchain-based method for the secure storage of patient records In ONC/NIST Use of Blockchain for Healthcare and Research Workshop. In ONC/NIST Use of Blockchain for Healthcare and Research Workshop (2837). Maryland: Gaithersburg.

Kuo, T.-T., Kim, H.-E., \& Ohno-Machado, L. (2017). Blockchain distributed ledger technologies for biomedical and health care applications. fournal of The American Medical Informatics Association, 1211-1220.

Legal Issues and Regulatory Developments. (2018). Review of Maritime Transport: UNCTAD. Retrieved from: https://unctad.org/en/PublicationChapters/ rmt2017ch5_en.pdf

List25. (2019, March 10). Retrieved from: https://list25.com/25-alarming-factsabout-organ-trafficking

Millet, J. (2019, 04 22). NewsBTC. Retrieved from: http:/ /www.newsbtc.com

Nakamoto, S. (2019, May 15). Bitcoin: A Peer-to-Peer Cash System. Retrieved from: https://nakamotoinstitute.org/bitcoin

Ojo, A. E. (n.d.). Blockchain as a next generation government information infrastructure: a review of initiatives in D5 countries. In Government 3.0-Next Generation Government Technology Infrastructure and Services. Springer, Cham, 283-298.

Shahbaz Pervez, M. M. (2018). Protective Measures For Security \& Privacy In Cyber Era Of Cloud Computing. International Conference on Electrical, Electronics, Computers, Communication, Mechanical and Computing. Villore: IEEE.

Shen, B. U. (2019). MedChain: Efficient Healthcare Data Sharing via Blockchain. Applied Sciences, 9.

Shimazono, Y. (2019). The state of the international organ trade: a provisional picture based on integration of available information. Bulletin of the World Health Organization, 85(12). Retrieved from: https://www.who.int/bulletin/ volumes/85/12/06-039370/en/

Snow, P. E., Deery, B., Kirby, P. \& Johnston, D. (2015). Factom ledger by consensus. 
The National Heath Gare Anti-Fraud Association. (2018, 11 13). Retrieved from: https://www.bcbsm.com/health-care-fraud/fraud-statistics.html

Waqas. (2019). Towards a Gateway-based Context-Aware and Self-Adaptive Security Management Model for IoT-based eHealth Systems. International fournal of Advanced Computer Science and Applications (IFACSA), 10.

Williams-Grut Technology Behind Bitcoin. (2019, january 04). 